[Chem. Pharm. Bull.

35(1) $\quad 90-96 \quad(1987)$

\title{
Synthesis and Reactions of 2,3-Dihydrothiazolo[3,2-a]pyrimidine Derivatives
}

\author{
Toshio Kinoshita, ${ }^{*}$ Yasuji Ueshima, Kouji Saitoh, \\ YaSUfumi Yoshida, and Sunao FuruKawa \\ Faculty of Pharmaceutical Sciences, Nagasaki University, \\ 1-14 Bunkyo-machi, Nagasaki 852, Japan
}

(Received June 9, 1986)

\begin{abstract}
The reaction of 2-amino-2-thiazoline (1) with acetylene carboxylates (2) afforded 5-substituted 2,3-dihydro-7 $H$-thiazolo[3,2-a]pyrimidin-7-ones (3). 7-Bromomethyl-2,3-dihydro-5 $\mathrm{H}$-thiazolo[3,2-a]pyrimidin-5-one (10) was obtained by the reaction of 1 with $\gamma$-bromoacetoacetyl bromide. Treatment of 10 with $N$-bromosuccinimide provided the 6-bromo compound (12). The 7bromomethyl compounds (10 and 12) were converted to 7-morpholinomethyl derivatives by treatment with morpholine. When 3a (unsubstituted-), 3b (5-ethoxycarbonyl-), and 3c (5hydroxymethyl-) were treated with $5 \%$ hydrochloric acid, covalent hydration occurred across the 5,6-carbon-carbon bond, giving 5-hydroxy-2,3,5,6-tetrahydro-7 $H$-thiazolo[3,2-a]pyrimidin-7-one derivatives (16a, 16b, and 16c). On the other hand, in the case of 11 (7-methyl-) and 17 (5-phenyl-), the dihydrothiazole ring was cleaved to give 3(and 1)-(2-mercaptoethyl)-6-methyl(and phenyl)2,4(1H,3H)-pyrimidinedione (19 and $\mathbf{1 8})$.
\end{abstract}

Keywords _-dihydrothiazolo[3,2-a]pyrimidine; $\gamma$-bromoacetoacetyl bromide; 2-amino-2thiazoline; covalent hydration; ring cleavage; bromination; cyclization; acetylene carboxylate

Various syntheses have been reported of 2,3-dihydrothiazolo[3,2-a]pyrimidine derivatives, some of which have analgesic, antithrombic and antiinflammatory activities. The compounds have been synthesized in two ways, when classified in terms of the starting materials. One involves cyclization of 2-thiouracil with 1,2-dibromoethane, ${ }^{1}{ }^{\prime}$ ethyl $\gamma$-chloroacetoacetate ${ }^{2)}$ or ethyl chloroacetate, ${ }^{3)}$ or intramolecular cyclization of 1-(2-hydroxyethyl)-thiouracil with methanesulfonyl chloride ${ }^{4)}$ or of 1 -allylthiouracil with iodine. ${ }^{5)}$ The other consists of the reaction of 2-amino-2-thiazoline (1) with diketene, ${ }^{6)}$ acetoacetic esters, ${ }^{7)}$ ethoxymethylenemalonic esters, $\left.{ }^{1 b}\right)$ ethoxymethylenecyanoacetamide ${ }^{8}{ }^{8}$ acetylene carboxylic ester, ${ }^{9)}$ ethyl malonyl chloride ${ }^{10)}$ or diethyl malonate. ${ }^{11)}$ In this paper we describe the synthesis of 2,3-dihydrothiazolo[3,2-a]pyrimidine derivatives from 2-amino-2-thiazoline (1) as a starting material, and some reactions of the thiazolopyrimidines.

The reaction of 2-amino-2-thiazoline (1) with ethyl propiolate $(2 \mathrm{a}, \mathrm{R}=\mathrm{H})$ and diethyl acetylenedicarboxylate $(\mathbf{2 b}, \mathrm{R}=\mathrm{COOEt})$ gave unsubstituted 2,3-dihydro-7H-thiazolo[3,2a]pyrimidin-7-one (3a) and 2,3-dihydro-5-ethoxycarbonyl-7 $\mathrm{H}$-thiazolo[3,2-a]pyrimidin-7-one (3b). Similarly, the 5-hydroxymethyl compound (3c) was obtained in $62.0 \%$ yield by treatment with ethyl $\gamma$-hydroxytetrolate $(\mathbf{2 c})^{12)}$ in ethanol. The structures of the above products $(\mathbf{3 a}, \mathbf{3 b}$, and $3 c$ ) are supported by the infrared (IR) and ultraviolet (UV) spectral data. ${ }^{1 b}$

Compound 3b was converted to the carboxamide (4) by reaction with ethanolic ammonia in a flask sealed by a stopcock at room temperature. The reaction of $3 \mathbf{c}$ with acetic anhydride afforded the corresponding acetoxy compound (5). For the preparation of the 5-halomethyl compounds (8 and $\mathbf{1 0}^{\prime}$ ), $3 \mathrm{c}$ was treated with thionyl chloride, phosphorus oxychloride or phosphorus tribromide under various conditions; however, no reaction occurred and the starting material was recovered.

6-Hydroxymethyl-2-thioxo-1,3-thiazin-4-one $(6)^{12)}$ was converted to 2,3-dihydro-1-(2- 

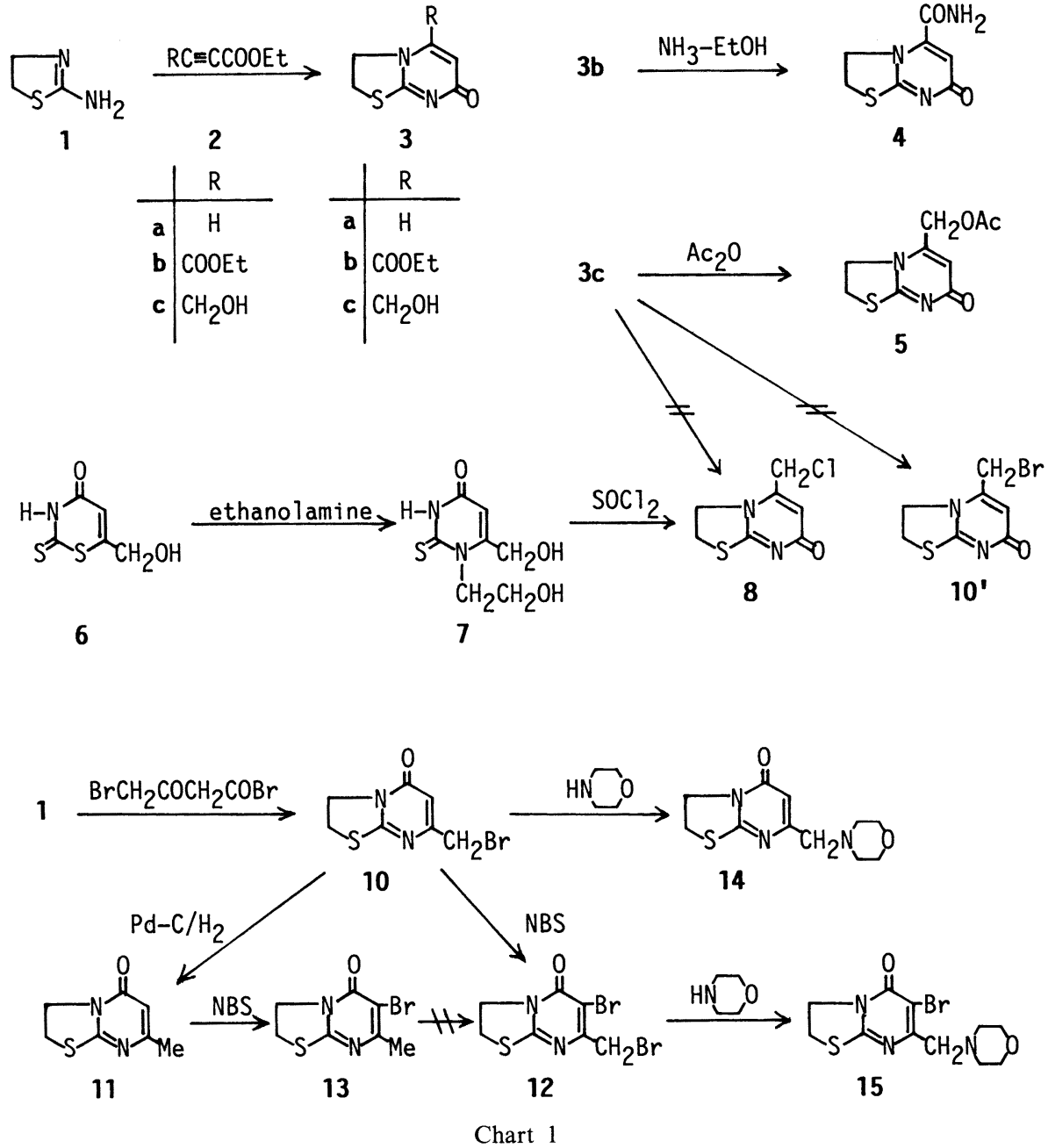

hydroxyethyl)-6-hydroxymethyl-2-thioxo-4(1H)-pyrimidinone (7) by reaction with 2-aminoethanol. In order to prepare 1-(2-chloroethyl)-6-chloromethyl-2-thioxo-4(3H)-pyrimidinone, 7 was treated with thionyl chloride, but the cyclized product 8 was obtained.

It has been reported that the reaction of acid chlorides with 2-aminoethiazole or 2amino-2-thiazoline (1) gives 2-acylaminothiazole ${ }^{13)}$ or 2-acylamino-2-thiazoline. ${ }^{12)}$ Therefore we expected that the reaction of $\gamma$-bromoacetoacetyl bromide $(9)^{14)}$ with 1 would afford $2-(\gamma-$ bromoacetoacetylamino)-2-thiazoline, followed by ring closure to yield 5-bromomethyl-2,3dihydro-7H-thiazolo[3,2-a]pyrimidin-7-one $\left(\mathbf{1 0}^{\prime}\right)$. However, we found that the product is 7 bromomethyl-2,3-dihydro-5H-thiazolo[3,2-a]pyrimidin-5-one (10). The structure of $\mathbf{1 0}$ was confirmed by IR and UV spectral data and chemical evidence. Compound 10 was converted to 7 -methyl-2,3-dihydro-5H-thiazolo[3,2-a]pyrimidin-5-one $(\mathbf{1 1})^{16,6)}$ by catalytic hydrogenolysis over palladium-carbon catalyst.

With $\mathrm{N}$-bromosuccinimide in ethanol, 10 was converted to the corresponding 6-bromo compound 12. Similarly, 11 was converted to the 6-bromo compound 13 by treatment with $N$ bromosuccinimide or bromine in acetic acid. ${ }^{1 b)}$ The position of the bromine substituent was determined from the proton nuclear magnetic resonance $\left({ }^{1} \mathrm{H}-\mathrm{NMR}\right)$ spectra of 12 and 13, in which no olefinic protons were not observed. It is well known that 5-bromo-1,3-disubstituted- 

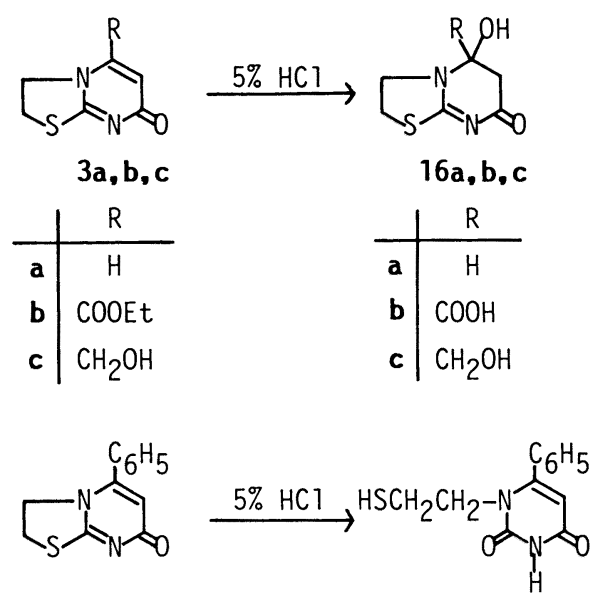

17<smiles>Cc1cc(=O)n2c(n1)SCC2</smiles>

11

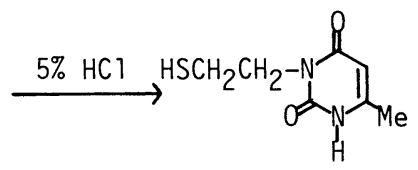

19<smiles>CCCCn1c(CC)cc(=O)n(CCC)c1=O</smiles>

20

Chart 2

6-methyl-2,4(1H,3H)-pyrimidinediones are converted to the 6-bromomethyl derivatives by reaction with bromine in acetic acid. ${ }^{15)}$ However, in the case of $\mathbf{1 3}$, no reaction occurred under these conditions. The reaction of the 7-bromomethyl compound (10 and 12) with morpholine yielded the corresponding 7-morpholinomethyl derivatives (14 and 15).

For the preparation of 1-(2-mercaptoethyl)-6-hydroxymethyl-2,4(1 H,3H)-pyrimidinedione, $3 \mathbf{c}$ was treated with $5 \%$ hydrochloric acid according to Falch and Natvig. ${ }^{16)}$ The product (16) was found to have the molecular formula $\mathrm{C}_{7} \mathrm{H}_{10} \mathrm{~N}_{2} \mathrm{O}_{3}$ by elemental microanalysis, but the UV spectrum showed no absorption maximum and was different from those of 2,4$(1 \mathrm{H}, 3 \mathrm{H})$-pyrimidinedione derivatives. ${ }^{17)}$

The carbon-13 nuclear magnetic resonance $\left({ }^{13} \mathrm{C}-\mathrm{NMR}\right)$ spectrum of $\mathbf{1 6} \mathbf{b}$ indicated the presence of three methylene groups at $\delta 48.93,40.80$, and 28.77. The former two signals are assignable to the carbons of the dihydrothiazole ring by comparison with those of the starting material (3b). Further structural assignment was possible from the ${ }^{1} \mathrm{H}-\mathrm{NMR}(270 \mathrm{MHz})$ spectrum, which exhibited signals due to one isolated methylene moiety at $\delta 3.70$ and 3.18 as doublets, complicated ethylene signals at $\delta 4.32,4.13$, and 3.5-3.3 and a characteristic broad singlet signal at $\delta 9.48$ due to a hydroxyl group.

The structure of $\mathbf{1 6} \mathbf{b}$, based on the above spectral data, was assumed to 5-carboxy-5hydroxy-2,3,5,6-tetrahydro-7H-thiazolo[3,2-a]pyrimidin-7-one, which corresponded to covalent hydration across the 5,6-carbon-carbon double bond of $\mathbf{3 b}$. Similarly, 3a and $\mathbf{3 c}$ were converted to the covalent hydration products $\mathbf{1 6 a}$ and 16c. However, under the same reaction conditions, no covalent hydration was observed in the case of compounds 17 and 11; instead, the dihydrothiazole ring was cleaved to afford 18 and 19, as described by Falch and Natvig. ${ }^{16}$ ) The UV spectra of the reaction products (18 and 19) showed absorption maxima at 275 and $264 \mathrm{~nm}$, and the IR spectra showed SH stretching absorptions at 2530 and $2555 \mathrm{~cm}^{-1}$. The 
${ }^{1} \mathrm{H}-\mathrm{NMR}$ spectrum of $\mathbf{1 8}$ showed signals at $\delta 1.48,2.80$, and 4.14 due to $\mathrm{NCH}_{2} \mathrm{CH}_{2} \mathrm{SH}$ and $\delta 5.91$ due to ring proton. Similarly, 19 exhibited signals at $\delta 1.19,2.64$, and 3.83 due to $\mathrm{NCH}_{2} \mathrm{CH}_{2} \mathrm{SH}$ and $\delta 5.63$ due to a ring proton in the ${ }^{1} \mathrm{H}-\mathrm{NMR}$ spectrum. On the basis of the above data, the structures of $\mathbf{1 8}$ and $\mathbf{1 9}$ were concluded to be 1-(2-mercaptoethyl)-6-phenyl2,4-(1H,3H)-pyrimidinedione and 3-(2-mercaptoethyl)-6-methyl-2,4-(1H,3H)-pyrimidinedione, respectively. When 11 was treated with $5 \%$ hydrochloric acid for $7 \mathrm{~h}$, an alternative product 20 was obtained which showed an absorption maximum at $263 \mathrm{~nm}$ in the UV spectrum (similar to that of 19). The ${ }^{1} \mathrm{H}-\mathrm{NMR}$ spectrum exhibited two double-doublet signals due to an ethylene group ( $\delta 4.33$ and 2.97$)$ and no peak due to an SH group. Furthermore, fast atom bombardment mass spectrometry (FAB-MS) showed the hydrogenated molecular ion peak $\left[(\mathrm{M}+1)^{+}\right]$at $m / z 371$. Therefore, 20 was concluded to be 3,3'-bis[6methyl-1,2,3,4-tetrahydro-2,4-dioxopyrimidinyl]diethyldisulfide. When 19 was treated with iodine in ethanol, $\mathbf{2 0}$ was obtained in good yield.

\section{Experimental}

Melting points reported here are uncorrected. IR spectra were recorded on JASCO IRA-2 and IR-810 spectrophotometer. UV spectra were recorded in ethanol on a Hitachi 323 spectrophotometer. The NMR spectra were obtained on Hitachi R-600 $\left(60 \mathrm{MHz}\right.$, for $\left.{ }^{1} \mathrm{H}\right)$, JEOL JNM FX-90Q $\left(90 \mathrm{MHz}\right.$ for ${ }^{1} \mathrm{H}$ and $22.5 \mathrm{MHz}$ for $\left.{ }^{13} \mathrm{C}\right)$ and JEOL JNM GX-270 $\left(270 \mathrm{MHz}\right.$, for $\left.{ }^{1} \mathrm{H}\right)$ spectrometers. Chemical shifts are reported in ppm $(\delta)$ relative to tetramethylsilane as an internal standard. Mass spectra were obtained on a JEOL JMS-DX-303 spectrometer by the electron impact $(\mathrm{EI})$ or fast atom bombardment $(\mathrm{FAB})$ ionization method.

2,3-Dihydro-7H-thiazolo[3,2-a]pyrimidin-7-one (3a) - A solution of 2-amino-2-thiazoline (1) $(0.51 \mathrm{~g}$, $5.0 \mathrm{mmol})$ and ethyl propiolate $(\mathbf{2 a})(0.5 \mathrm{~g}, 5.0 \mathrm{mmol})$ in $\mathrm{EtOH}(20 \mathrm{ml})$ was refluxed for $9 \mathrm{~h}$. The reaction mixture was concentrated to dryness in vacuo and the residue was washed with ether. The insoluble material was crystallized from $\mathrm{MeOH}$, giving $0.53 \mathrm{~g}\left(68.8 \%\right.$ ) of colorless needles, mp $225 \mathrm{C}$ (lit. $\left.{ }^{1 b)} \mathrm{mp} 227-228 \mathrm{C}\right) .{ }^{1} \mathrm{H}-\mathrm{NMR}\left(\mathrm{CDCl}_{3}\right) \delta: 7.27(1 \mathrm{H}$, d, $J=7.8 \mathrm{~Hz}, \mathrm{C}(5)-\mathrm{H}), 6.02(1 \mathrm{H}, \mathrm{d}, J=7.8 \mathrm{~Hz}, \mathrm{C}(6)-\mathrm{H}), 4.34\left(2 \mathrm{H}, \mathrm{t}, J=6.8 \mathrm{~Hz}, \mathrm{~N}-\mathrm{CH}_{2}\right), 3.50(2 \mathrm{H}, \mathrm{t}, J=6.8 \mathrm{~Hz}$, $\left.\mathrm{S}-\mathrm{CH}_{2}\right)$. MS $m / z: 154\left(\mathrm{M}^{+}\right)$.

2,3-Dihydro-5-ethoxycarbonyl-7H-thiazolo[3,2-a]pyrimidin-7-one (3b) - A solution of $\mathbf{1}(0.5 \mathrm{~g}, 5.0 \mathrm{mmol})$ and diethyl acetylenedicarboxylate (2b) $(0.85 \mathrm{~g}, 5.0 \mathrm{mmol})$ in EtOH $(20 \mathrm{ml})$ was refluxed for $3 \mathrm{~h}$. The reaction mixture was concentrated to dryness in vacuo, and the residue was recrystallized from AcOEt, giving $0.61 \mathrm{~g}(61.0 \%)$ of colorless needles, mp 121-122 C. Anal. Calcd for $\mathrm{C}_{9} \mathrm{H}_{10} \mathrm{~N}_{2} \mathrm{O}_{3} \mathrm{~S}$ : C, 47.79; H, 4.42; N, 12.39; S, 14.16. Found: C, 47.53; H, 4.43; N, 12.43; S, 14.27. IR (KBr): 1724, $1625(\mathrm{C}=\mathrm{O}) \mathrm{cm}^{-1}$. UV $\lambda_{\max }^{\text {ethanol }} \mathrm{nm}(\log \varepsilon): 239(4.34), 290(3.68) .{ }^{1} \mathrm{H}-\mathrm{NMR}$ $\left(\mathrm{CDCl}_{3}\right) \delta: 6.70(1 \mathrm{H}, \mathrm{s}, \mathrm{C}(6)-\mathrm{H}), 4.80\left(2 \mathrm{H}, \mathrm{t}, J=7.5 \mathrm{~Hz}, \mathrm{~N}-\mathrm{CH}_{2}\right), 4.38\left(2 \mathrm{H}, \mathrm{q}, J=7.2 \mathrm{~Hz}, \mathrm{C}_{2} \mathrm{CH}_{3}\right), 3.48(2 \mathrm{H}, \mathrm{t}, J=$ $\left.7.5 \mathrm{~Hz}, \mathrm{~S}-\mathrm{CH}_{2}\right), 1.39\left(3 \mathrm{H}, \mathrm{t}, J=7.2 \mathrm{~Hz}, \mathrm{CH}_{2} \mathrm{CH}_{3}\right)$. FAB-MS $m / z: 227\left[(\mathrm{M}+1)^{+}\right]$.

2,3-Dihydro-5-hydroxymethyl-7H-thiazolo[3,2-a]pyrimidin-7-one (3c) A solution of $\mathbf{1}(0.25 \mathrm{~g}, 2.5 \mathrm{mmol})$ and ethyl $\gamma$-hydroxytetrolate $(2 \mathrm{c})(0.32 \mathrm{~g}, 2.5 \mathrm{mmol})$ in $\mathrm{MeOH}(10 \mathrm{ml})$ was refluxed for $3 \mathrm{~h}$. The reaction mixture was concentrated to dryness in vacuo, and the residue was crystallized from $\mathrm{MeOH}$, giving $0.28 \mathrm{~g}(62.0 \%)$ of colorless needles, mp 267-269 C. Anal. Calcd for $\mathrm{C}_{7} \mathrm{H}_{8} \mathrm{~N}_{2} \mathrm{O}_{2} \mathrm{~S}: \mathrm{C}, 45.65 ; \mathrm{H}, 4.38 ; \mathrm{N}, 15.21 ; \mathrm{S}, 17.40$. Found: C, 45.79; H, 4.50; $\mathrm{N}, 15.14 ; \mathrm{S}, 17.32$. IR $(\mathrm{KBr}): 3210(\mathrm{OH}), 1632(\mathrm{C}=\mathrm{O}) \mathrm{cm}^{-1}$. UV $\lambda_{\text {max }}^{\text {ethanol }} \mathrm{nm}(\log \varepsilon): 232.5(4.45), c a .268(\mathrm{sh}, 4.00) .{ }^{1} \mathrm{H}-$ NMR (DMSO- $\left.d_{6}-\mathrm{CF}_{3} \mathrm{COOH}=1: 1\right): 6.27(1 \mathrm{H}, \mathrm{s}, \mathrm{C}(6)-\mathrm{H}), 5.48(1 \mathrm{H}, \mathrm{s}, \mathrm{OH}), 4.78\left(2 \mathrm{H}, \mathrm{dd}, J=7.6,8.3 \mathrm{~Hz}, \mathrm{~N}-\mathrm{CH}_{2}\right)$, $4.74\left(2 \mathrm{H}, \mathrm{s}, \mathrm{O}-\mathrm{CH}_{2}\right), 3.85\left(2 \mathrm{H}, \mathrm{dd}, J=7.6,8.3 \mathrm{~Hz}, \mathrm{~S}-\mathrm{CH}_{2}\right) . \mathrm{FAB}-\mathrm{MS} m / z: 185\left[(\mathrm{M}+1)^{+}\right]$.

5-Carbamoyl-2,3-dihydro-7 $H$-thiazolo[3,2-a]pyrimidin-7-one (4) A solution of $3 \mathrm{~b}(0.1 \mathrm{~g}, 0.44 \mathrm{mmol})$ in $\mathrm{NH}_{3}-\mathrm{EtOH}$ (saturated, $20 \mathrm{ml}$ ) was sealed in a flask with a stopcock and allowed to stand at room temperature overnight. The separated crystalline mass was collected and recrystallized from $\mathrm{MeOH}$, giving $70 \mathrm{mg}(61.0 \%)$ of colorless needles, mp 289-290 C. Anal. Calcd for $\mathrm{C}_{7} \mathrm{H}_{7} \mathrm{~N}_{3} \mathrm{O}_{2} \mathrm{~S}: \mathrm{C}, 42.63 ; \mathrm{H}, 3.58 ; \mathrm{N}, 21.30 ; \mathrm{S}, 16.26$. Found: $\mathrm{C}$, 42.33; H, 3.64; N, 21.08; S, 16.04. IR ( $(\mathrm{KBr}): 3440,3320\left(\mathrm{NH}_{2}\right), 1695(\mathrm{C}=\mathrm{O}) \mathrm{cm}^{-1} . \mathrm{UV} i_{\max }^{\text {ethanol }} \mathrm{nm}(\log \varepsilon): 236(4.42)$, $286(\mathrm{sh}, 3.88) .{ }^{1} \mathrm{H}-\mathrm{NMR}$ (DMSO- $\left.d_{6}-\mathrm{CF}_{3} \mathrm{COOH}=1: 1\right) \delta: 8.41(1 \mathrm{H}, \mathrm{brs}, \mathrm{NH}), 8.00(1 \mathrm{H}, \mathrm{brs}, \mathrm{NH}), 6.77(1 \mathrm{H}, \mathrm{s}$, $\mathrm{C}(6)-\mathrm{H}), 4.87\left(2 \mathrm{H}, \mathrm{t}, J=7.9 \mathrm{~Hz}, \mathrm{~N}-\mathrm{CH}_{2}\right), 3.74\left(2 \mathrm{H}, \mathrm{t}, J=7.9 \mathrm{~Hz}, \mathrm{~S}-\mathrm{CH}_{2}\right) . \mathrm{FAB}-\mathrm{MS} m / z: 198\left[(\mathrm{M}+1)^{+}\right]$.

5-Acetoxymethyl-2,3-dihydro-7 $\boldsymbol{H}$-thiazolo[3,2-a]pyrimidin-7-one (5)-A mixture of $\mathbf{3 c}(1.8 \mathrm{~g}, 9.8 \mathrm{mmol})$ and acetic anhydride $(30 \mathrm{ml})$ was refluxed for $3 \mathrm{~h}$. The reaction mixture was concentrated to dryness in vacuo, and the residue was crystallized from acetone, giving $2.0 \mathrm{~g}(90.3 \%)$ of colorless needles, mp $137-138{ }^{\circ} \mathrm{C}$. Anal. Calcd for $\mathrm{C}_{9} \mathrm{H}_{10} \mathrm{~N}_{2} \mathrm{O}_{3} \mathrm{~S}: \mathrm{C}, 47.77 ; \mathrm{H}, 4.46 ; \mathrm{N}, 12.38 ; \mathrm{S}, 14.17$. Found: C, 47.84; H, 4.44; N, 12.49; S, 13.88. IR (KBr): 1740,1630 $(\mathrm{C}=\mathrm{O}) \mathrm{cm}^{-1} . \mathrm{UV} i_{\max }^{\mathrm{ethanol}} \mathrm{nm}(\log \varepsilon): 233(4.38), c a .268(\mathrm{sh}, 3.82) .{ }^{1} \mathrm{H}-\mathrm{NMR}\left(\mathrm{CDCl}_{3}\right) \delta: 6.00(1 \mathrm{H}, \mathrm{s}, \mathrm{C}(6)-\mathrm{H}), 4.58$ $\left(2 \mathrm{H}, \mathrm{s}, \mathrm{O}-\mathrm{CH}_{2}\right), 4.38\left(2 \mathrm{H}, \mathrm{dd}, J=6.8,7.9 \mathrm{~Hz}, \mathrm{~N}-\mathrm{CH}_{2}\right), 3.49\left(2 \mathrm{H}, \mathrm{dd}, J=6.8,7.9 \mathrm{~Hz}, \mathrm{~S}-\mathrm{CH}_{2}\right), 2.17\left(3 \mathrm{H}, \mathrm{s}, \mathrm{CO}-\mathrm{CH}_{3}\right)$. FAB-MS $m / z: 227\left[(\mathrm{M}+1)^{+}\right]$.

2,3-Dihydro-1-(2-hydroxyethyl)-6-hydroxymethyl-2-thioxo-4(1 H)-pyrimidinone (7)-A solution of $6^{12)}(2.4 \mathrm{~g}$, 
$13.7 \mathrm{mmol})$ and ethanolamine $(1.6 \mathrm{~g}, 26.5 \mathrm{mmol})$ in $\mathrm{EtOH}(10 \mathrm{ml})$ was refluxed for $1 \mathrm{~h}$. After cooling, the mixture was acidified with $10 \% \mathrm{HCl}$, and left to stand in a refrigerator overnight. After concentration, the separated crystalline mass was collected and recrystallized from $\mathrm{EtOH}$, giving $1.05 \mathrm{~g}(37.9 \%)$ of colorless prisms, mp $199-200^{\circ} \mathrm{C}$. Anal. Calcd for $\mathrm{C}_{7} \mathrm{H}_{10} \mathrm{~N}_{2} \mathrm{O}_{3} \mathrm{~S}: \mathrm{C}, 41.58 ; \mathrm{H}, 4.98 ; \mathrm{N}, 13.85 ; \mathrm{S}, 15.85$. Found: $\mathrm{C}, 41.45 ; \mathrm{H}, 4.99 ; \mathrm{N}, 13.71 ; \mathrm{S}, 15.78$. IR (KBr): $1650(\mathrm{C}=\mathrm{O}) \mathrm{cm}^{-1}$. UV $i_{\max }^{\text {ethanol }} \mathrm{nm}(\log \varepsilon): 221(4.25), 278(4.21) .{ }^{1} \mathrm{H}-\mathrm{NMR}\left(\mathrm{CDCl}_{3}\right) \delta: 12.44(1 \mathrm{H}, \mathrm{brs}, \mathrm{NH}), 5.98$ $(1 \mathrm{H}, \mathrm{s}$, ring $), 4.51(2 \mathrm{H}$, br s, C(6)-CH $), 4.29\left(2 \mathrm{H}, \mathrm{t}, J=5.5 \mathrm{~Hz}, \mathrm{~N}-\mathrm{CH}_{2}\right), 3.74\left(2 \mathrm{H}, \mathrm{q}, J=5.5 \mathrm{~Hz}, \mathrm{~S}-\mathrm{CH}_{2}\right)$.

5-Chloromethyl-2,3-dihydro-7 $\boldsymbol{H}$-thiazolo[3,2-a]pyrimidin-7-one (8)-A mixture of $7(0.3 \mathrm{~g}, 14.9 \mathrm{mmol})$ and thionyl chloride $(4.9 \mathrm{~g}, 41.2 \mathrm{mmol})$ with 2 drops of pyridine as a catalyst was stirred at room temperature for $5 \mathrm{~h}$. The reaction mixture was poured onto ice, then neutralized with $5 \% \mathrm{NaHCO}_{3}$. The mixture was extracted with $\mathrm{CHCl}_{3}$, and the extract was dried over $\mathrm{Na}_{2} \mathrm{SO}_{4}$. After evaporation of the solvent, the residue was crystallized from acetone, giving $50 \mathrm{mg}(16.7 \%)$ of colorless needles, mp $174-175^{\circ} \mathrm{C}$. Anal. Calcd for $\mathrm{C}_{7} \mathrm{H}_{7} \mathrm{ClN}_{2} \mathrm{OS}$ : C, 41.49; $\mathrm{H}, 3.48 ; \mathrm{N}$, 13.82; S, 15.82. Found: C, 41.74; H, 3.68; N, 13.57; S, 15.74. UV $i_{\max }^{\text {ethanol }} \mathrm{nm}(\log \varepsilon): 234(4.40), c a .270(\mathrm{sh}, 3.85) .{ }^{1} \mathrm{H}-$ $\operatorname{NMR}\left(\mathrm{CDCl}_{3}\right) \delta: 6.30(1 \mathrm{H}, \mathrm{s}, \mathrm{C}(6)-\mathrm{H}), 4.59\left(2 \mathrm{H}, \mathrm{t}, J=7.5 \mathrm{~Hz}, \mathrm{~N}-\mathrm{CH}_{2}\right), 3.55\left(2 \mathrm{H}, \mathrm{t}, J=7.5 \mathrm{~Hz}, \mathrm{~S}-\mathrm{CH}_{2}\right), 3.06(2 \mathrm{H}, \mathrm{s}$, $\mathrm{CH}_{2}-\mathrm{Cl}$ ).

7-Bromomethyl-2,3-dihydro-5 $\boldsymbol{H}$-thiazolo[3,2-a]pyrimidin-5-one (10)_A solution of bromine $(6.4 \mathrm{~g}, 40 \mathrm{mmol})$ in $\mathrm{CCl}_{4}(15 \mathrm{ml})$ was added to a solution of diketene $(3.36 \mathrm{~g}, 40 \mathrm{mmol})$ in $\mathrm{CCl}_{4}(30 \mathrm{ml})$ under ice cooling with stirring, and the temperature was maintained for $2 \mathrm{~h}$. The reaction mixture was added to a solution of $1(2.04 \mathrm{~g}, 20 \mathrm{mmol}) \mathrm{in}$ $\mathrm{CHCl}_{3}(30 \mathrm{ml})$ under ice cooling with stirring, and the whole was maintained at the same temperature for $2 \mathrm{~h}$, then at room temperature for $3 \mathrm{~h}$. The separated crystalline mass was collected and recrystallized from $\mathrm{MeOH}$, giving $2.4 \mathrm{~g}$ $(36.5 \%)$ of brown prisms, mp $299-300 \mathrm{C}$ ( $\mathrm{HBr}$ salt). These crystals were dissolved in $5 \% \mathrm{NaHCO}_{3}$, and the solution was extracted with $\mathrm{CHCl}_{3}$. The extract was dried over $\mathrm{MgSO}_{4}$. After evaporation of the solvent, the residue was crystallized from $\mathrm{MeOH}$, giving $0.9 \mathrm{~g}\left(18.2^{\circ}\right.$ ) of colorless needles, mp $98-99^{\circ} \mathrm{C}$. Anal. Calcd for $\mathrm{C}_{7} \mathrm{H}_{7} \mathrm{BrN}_{2} \mathrm{OS}: \mathrm{C}_{\text {, }}$ $34.02 ; \mathrm{H}, 2.85 ; \mathrm{Br}, 32.34 ; \mathrm{N}, 11.34 ; \mathrm{S}, 12.97$. Found: C, 34.18; H, 2.87; Br, 31.87; N, 11.32; S, 12.97. IR (KBr): 1660 $(\mathrm{C}=\mathrm{O}) \mathrm{cm}^{-1}$. UV $\lambda_{\max }^{\mathrm{ethan}} \mathrm{nm}(\log \varepsilon): 233(3.94), 298(3.81) .{ }^{1} \mathrm{H}-\mathrm{NMR}\left(\mathrm{CDCl}_{3}\right) \delta: 6.25(1 \mathrm{H}, \mathrm{s}, \mathrm{C}(6)-\mathrm{H}), 4.47(2 \mathrm{H}, \mathrm{t}, J=$ $\left.7.5 \mathrm{~Hz}, \mathrm{~N}-\mathrm{CH}_{2}\right), 4.14\left(2 \mathrm{H}, \mathrm{s}, \mathrm{CH}_{2}-\mathrm{Br}\right), 3.47\left(2 \mathrm{H}, \mathrm{t}, J=7.5 \mathrm{~Hz}, \mathrm{~S}-\mathrm{CH}_{2}\right) . \mathrm{MS} m / z: 248$ and $246\left(\mathrm{M}^{+}\right)$.

Hydrogenolysis of 10 A mixture of $10(60 \mathrm{mg}, 0.24 \mathrm{mmol})$ and $5 \% \mathrm{Pd}-\mathrm{C}(50 \mathrm{mg})$ in EtOH $(20 \mathrm{ml})$ was stirred under a hydrogen atmosphere at room temperature for $8 \mathrm{~h}$. The reaction mixture was filtered, and the filtrate was concentrated to dryness in vacuo. The residue was treated with $5 \% \mathrm{NaHCO}_{3}$, then extracted with $\mathrm{CHCl}_{3}$. The extract was dried over $\mathrm{MgSO}_{4}$ and filtered. The filtrate was evaporated to dryness and the residue was crystallized from AcOEt, giving 11, $40 \mathrm{mg}\left(99.2 \%\right.$ ) of colorless needles, mp $126-127^{\circ} \mathrm{C}$ (lit. ${ }^{1 b)} \mathrm{mp} 127-128^{\circ} \mathrm{C}$ ).

6-Bromo-7-bromomethyl-2,3-dihydro-5H-thiazolo[3,2-a]pyrimidine-5-one (12)—A solution of 10 (247 mg, $1.00 \mathrm{mmol})$ and $\mathrm{N}$-bromosuccinimide $(200 \mathrm{mg}, 1.12 \mathrm{mmol})$ in EtOH $(20 \mathrm{ml})$ was refluxed for $9 \mathrm{~h}$. The reaction mixture was concentrated to dryness in vacuo, and the residue was crystallized from $\mathrm{MeOH}$, giving $200 \mathrm{mg}(61.3 \%)$ as colorless needles, mp 166-167 ${ }^{\circ}$. Anal. Calcd for $\mathrm{C}_{7} \mathrm{H}_{6} \mathrm{Br}_{2} \mathrm{~N}_{2} \mathrm{OS}$ : C, 25.79; H, 1.86; N, 8.59; Br, 49.02. Found: C, 25.96; H, 2.00; N, 8.57; Br, 48.57. IR (KBr): $1661(\mathrm{C}=\mathrm{O}) \mathrm{cm}^{-1}$. UV $\lambda_{\max }^{\text {ethan }} \mathrm{nm}(\log \varepsilon): 245(3.94), 317(3.94) .{ }^{1} \mathrm{H}-$ NMR $\left(\mathrm{CDCl}_{3}\right) \delta: 4.52\left(2 \mathrm{H}, \mathrm{t}, J=7.9 \mathrm{~Hz}, \mathrm{~N}-\mathrm{CH}_{2}\right), 4.39\left(2 \mathrm{H}, \mathrm{s}, \mathrm{CH}_{2}-\mathrm{Br}\right), 3.52\left(2 \mathrm{H}, \mathrm{t}, J=7.9 \mathrm{~Hz}, \mathrm{~S}-\mathrm{CH}_{2}\right)$. MS $m / z$ : 328,326 , and $324\left(\mathrm{M}^{+}\right)$.

6-Bromo-2,3-dihydro-7-methyl-5H-thiazolo[3,2-a]pyrimidin-5-one (13) - a) A solution of 11 (168 mg, 1.00 $\mathrm{mmol})$ and $N$-bromosuccinimide $(200 \mathrm{mg}, 1.12 \mathrm{mmol})$ in EtOH $(20 \mathrm{ml})$ was refluxed for $4.5 \mathrm{~h}$. The reaction mixture was ice-cooled and the separated crystalline mass was collected, then crystallized from $\mathrm{MeOH}$, giving $100 \mathrm{mg}(40.0 \%)$ of colorless prisms, mp 209-210 C. Anal. Calcd for $\mathrm{C}_{7} \mathrm{H}_{7} \mathrm{BrN}_{2} \mathrm{OS}: \mathrm{C}, 34.02 ; \mathrm{H}, 2.86 ; \mathrm{Br}, 32.34 ; \mathrm{N}, 11.34 ; \mathrm{S}, 12.97$. Found: C, 33.90; H, 2.93; Br, 32.34; N, 11.26; S, 13.06. IR $(\mathrm{KBr}): 1662(\mathrm{C}=\mathrm{O}) \mathrm{cm}^{-1}$. UV $\lambda_{\max }^{\text {ethanol }} \mathrm{nm}(\log \varepsilon): 250$ (3.78), 304 (3.99). ${ }^{1} \mathrm{H}-\mathrm{NMR}\left(\mathrm{CDCl}_{3}\right) \delta: 4.51\left(2 \mathrm{H}, \mathrm{t}, J=7.5 \mathrm{~Hz}, \mathrm{~N}-\mathrm{CH}_{2}\right), 3.50\left(2 \mathrm{H}, \mathrm{t}, J=7.5 \mathrm{~Hz}, \mathrm{~S}-\mathrm{CH}_{2}\right), 2.43(3 \mathrm{H}, \mathrm{s}$, $\left.\mathrm{CH}_{3}\right)$. MS $m / z$ : 248 and $246\left(\mathrm{M}^{+}\right)$.

b) A solution of bromine $(0.3 \mathrm{ml})$ in acetic acid $(1 \mathrm{ml})$ was added to a solution of $11(340 \mathrm{mg}, 2.02 \mathrm{mmol})$ in acetic acid $(7 \mathrm{ml})$ with stirring; a reddish brown crystalline mass immediately separated. The mixture was heated at 90 $95^{\circ} \mathrm{C}$ for $2 \mathrm{~h}$. After cooling, the separated crystalline mass was collected and recrystallized from $\mathrm{MeOH}$, giving $340 \mathrm{mg}(68.1 \%)$ of colorless prisms, mp $209-210{ }^{\circ} \mathrm{C}$.

2,3-Dihydro-7-morpholinomethyl-5 $\boldsymbol{H}$-thiazolo[3,2-a]pyrimidin-5-one (14)-A solution of 10 (1.0 g, $4.0 \mathrm{mmol})$ and morpholine $(0.7 \mathrm{~g}, 8.0 \mathrm{mmol})$ in $\mathrm{EtOH}(80 \mathrm{ml})$ was refluxed for $6 \mathrm{~h}$. The reaction mixture was concentrated to dryness in vacuo. The residue was crystallized from $\mathrm{MeOH}$, giving $0.9 \mathrm{~g}(87.9 \%)$ of colorless plates, mp $163-164 \mathrm{C}$. Anal. Calcd for $\mathrm{C}_{11} \mathrm{H}_{15} \mathrm{~N}_{3} \mathrm{O}_{2} \mathrm{~S}: \mathrm{C}, 52.15 ; \mathrm{H}, 5.97 ; \mathrm{N}, 16.59 ; \mathrm{S}, 12.66$. Found: C, 51.94; H, 6.00; N, 16.55; S, 12.72. IR $(\mathrm{KBr}): 1675,1653(\mathrm{C}=\mathrm{O}) \mathrm{cm}^{-1}$. UV $\lambda_{\max }^{\text {ethanol }} \mathrm{nm}(\log \varepsilon): 230(3.89), 292(3.85) .{ }^{1} \mathrm{H}-\mathrm{NMR}\left(\mathrm{CDCl}_{3}\right) \delta: 6.29(1 \mathrm{H}, \mathrm{s}, \mathrm{C}(6)-$ $\mathrm{H}), 4.47\left(2 \mathrm{H}, \mathrm{t}, J=7.9 \mathrm{~Hz}, \mathrm{~N}-\mathrm{CH}_{2}\right), 3.74\left(4 \mathrm{H}, \mathrm{m}, 2 \times \mathrm{O}-\mathrm{CH}_{2}\right), 3.46\left(2 \mathrm{H}, \mathrm{t}, J=7.9 \mathrm{~Hz}, \mathrm{~S}-\mathrm{CH}_{2}\right), 3.33(2 \mathrm{H}, \mathrm{s}, \mathrm{C}(7)-$ $\left.\mathrm{CH}_{2}\right), 2.52\left(4 \mathrm{H}, \mathrm{m}, 2 \times \mathrm{N}-\mathrm{CH}_{2}\right)$. MS $m / z: 253\left(\mathrm{M}^{+}\right)$.

6-Bromo-2,3-dihydro-7-morpholinomethyl-5H-thiazolo[3,2-a]pyrimidin-5-one (15)_-A solution of $12(652 \mathrm{mg}$, $2.0 \mathrm{mmol})$ and morpholine $(348 \mathrm{mg}, 4.0 \mathrm{mmol})$ in $\mathrm{EtOH}(60 \mathrm{ml})$ was refluxed for $3 \mathrm{~h}$. The reaction mixture was concentrated, and the separated crystalline mass was collected and recrystallized from $\mathrm{MeOH}-\mathrm{AcOEt}$, giving $300 \mathrm{mg}$ $(45.2 \%)$ of colorless needles, mp $141-142^{\circ} \mathrm{C}$. Anal. Calcd for $\mathrm{C}_{11} \mathrm{H}_{14} \mathrm{BrN}_{3} \mathrm{O}_{2} \mathrm{~S}: \mathrm{C}, 39.77 ; \mathrm{H}, 4.25 ; \mathrm{N}, 12.65$. Found: $\mathrm{C}, 39.85 ; \mathrm{H}, 4.07 ; \mathrm{N}, 12.53$. IR $(\mathrm{KBr}): 1661(\mathrm{C}=\mathrm{O}) \mathrm{cm}^{-1}$. UV $\lambda_{\max }^{\text {ethan }} \mathrm{nm}(\log \varepsilon): 242(3.84), 308(3.94) .{ }^{1} \mathrm{H}-\mathrm{NMR}$ 
$\left(\mathrm{CDCl}_{3}\right) \delta: 4.52\left(2 \mathrm{H}, \mathrm{t}, J=7.8 \mathrm{~Hz}, \mathrm{~N}-\mathrm{CH}_{2}\right), 3.74\left(4 \mathrm{H}, \mathrm{m}, 2 \times \mathrm{O}-\mathrm{CH}_{2}\right), 3.61\left(2 \mathrm{H}, \mathrm{s}, \mathrm{C}(7)-\mathrm{CH}_{2}\right), 3.49(2 \mathrm{H}, \mathrm{t}, J=7.8 \mathrm{~Hz}$, $\left.\mathrm{S}-\mathrm{CH}_{2}\right), 2.60\left(4 \mathrm{H}, \mathrm{m}, 2 \times \mathrm{N}-\mathrm{CH}_{2}\right) . \mathrm{MS} m / z: 333$ and $331\left(\mathrm{M}^{+}\right)$.

5-Hydroxy-2,3,5,6-tetrahydro-7 $\mathbf{H}$-thiazolo[3,2-a]pyrimidin-7-one (16a)-A solution of 3a $(1.0 \mathrm{~g}, 6.49 \mathrm{mmol})$ in $5 \% \mathrm{HCl}(20 \mathrm{ml})$ was heated at $95-100{ }^{\circ} \mathrm{C}$ for $9 \mathrm{~h}$. The reaction mixture was concentrated to dryness in vacuo. Water was added to the residue, and the mixture was evaporated to dryness in vacuo. The crystalline residue was recrystallized from $\mathrm{H}_{2} \mathrm{O}$, giving $720 \mathrm{mg}(64.5 \%)$ of colorless prisms, mp $211-213^{\circ} \mathrm{C}$. Anal. Calcd for $\mathrm{C}_{6} \mathrm{H}_{8} \mathrm{~N}_{2} \mathrm{O}_{2} \mathrm{~S}: \mathrm{C}_{\text {, }}$ 41.85; H, 4.68; N, 16.27; S, 18.62. Found: C, 41.86; H, 4.48; N, 16.16; S, 18.81. IR (KBr): 3400, 3170 (OH), 1700 (br) $(\mathrm{C}=\mathrm{O}) \mathrm{cm}^{-1}$. UV: no absorption maximum. ${ }^{1} \mathrm{H}-\mathrm{NMR}\left(270 \mathrm{MHz}: \mathrm{CDCl}_{3}\right.$ and 3 drops of $\left.\mathrm{CF}_{3} \mathrm{COOH}\right) \delta: 9.23(1 \mathrm{H}, \mathrm{s}$, $\mathrm{OH}), 5.02(1 \mathrm{H}, \mathrm{dd}, J=4.4,12.6 \mathrm{~Hz}, \mathrm{C}(5)-\mathrm{H}), 4.16(1 \mathrm{H}, \mathrm{dt}, J=6.6,6.6,11.5 \mathrm{~Hz}, \mathrm{~N}-\mathrm{CH}), 3.85(1 \mathrm{H}$, quintet, $J=5.5,5.5$, $11.5 \mathrm{~Hz}, \mathrm{~N}-\mathrm{CH}), 3.3-3.15\left(2 \mathrm{H}, \mathrm{m}, \mathrm{S}-\mathrm{CH}_{2}\right), 3.14(1 \mathrm{H}, \mathrm{dd}, J=4.4,17.0 \mathrm{~Hz}, \mathrm{C}(6)-\mathrm{H}), 2.92(1 \mathrm{H}, \mathrm{dd}, J=12.6,17.0 \mathrm{~Hz}$, $\mathrm{C}(6)-\mathrm{H}) .{ }^{13} \mathrm{C}-\mathrm{NMR}$ (pyridine- $\left.d_{5}\right) \dot{\delta}: 169.29(\mathrm{~s}, \mathrm{C}(7$ or 1a)), $151.33(\mathrm{~s}, \mathrm{C}(1 \mathrm{la}$ or 7$)), 57.43(\mathrm{~d}, \mathrm{C}(5)), 48.82(\mathrm{t}, \mathrm{C}(3)), 38.44$ $(\mathrm{t}, \mathrm{C}(2)), 29.28(\mathrm{t}, \mathrm{C}(6))$. FAB-MS $m / z: 173\left[(\mathrm{M}+1)^{+}\right]$.

5-Carboxy-2,3,5,6-tetrahydro-5-hydroxy-7H-thiazolo[3,2-a]pyrimidin-7-one (16b) - A solution of $3 \mathrm{~b}(0.56 \mathrm{~g}$, $2.48 \mathrm{mmol})$ in $5 \% \mathrm{HCl}(6 \mathrm{ml})$ was heated at $90-95^{\circ} \mathrm{C}$ for $8 \mathrm{~h}$. The reaction mixture was worked up as described above, giving $0.4 \mathrm{~g}\left(74.7^{\circ}, \mathrm{H}_{2} \mathrm{O}\right.$ ), of colorless powder, mp $236^{\circ} \mathrm{C}$ (bub.). Anal. Calcd for $\mathrm{C}_{7} \mathrm{H}_{8} \mathrm{~N}_{2} \mathrm{O}_{4} \mathrm{~S}: \mathrm{C}, 38.88 ; \mathrm{H}$, 3.73; N, 12.96; S, 14.83. Found: C, 38.18; H, 3.66; N, 12.98; S, 14.88. IR (KBr): $3245(\mathrm{OH}), 1738,1720$ (sh), 1659 $(\mathrm{C}=\mathrm{O}) \mathrm{cm}^{-1}$. UV: no absorption maximum. ${ }^{1} \mathrm{H}-\mathrm{NMR}\left(270 \mathrm{MHz}: \mathrm{CDCl}_{3}\right.$ and 3 drops of $\left.\mathrm{CF}_{3} \mathrm{COOH}\right) \delta: 9.48(1 \mathrm{H}, \mathrm{s}$, $\mathrm{OH}), 4.32(1 \mathrm{H}, \mathrm{dt}, J=6.9,6.9,11.5 \mathrm{~Hz}, \mathrm{~N}-\mathrm{CH}), 4.13(1 \mathrm{H}, \mathrm{ddd}, J=5.0,6.9,11.5 \mathrm{~Hz}, \mathrm{~N}-\mathrm{CH}), 3.70(1 \mathrm{H}, \mathrm{d}, J=$ $17.0 \mathrm{~Hz}, \mathrm{C}(6)-\mathrm{H}), 3.5-3.3\left(2 \mathrm{H}, \mathrm{m}, \mathrm{S}-\mathrm{CH}_{2}\right), 3.18(1 \mathrm{H}, \mathrm{d}, J=17.0 \mathrm{~Hz}, \mathrm{C}(6)-\mathrm{H}) .{ }^{13} \mathrm{C}-\mathrm{NMR}$ (DMSO- $\left.d_{6}\right) \delta: 171.43(\mathrm{~s}$, $\mathrm{COOH}), 167.47$ (s, C(7 or 1a)), $149.43(\mathrm{~s}, \mathrm{C}(1 \mathrm{a}$ or 7$)), 67.08(\mathrm{~s}, \mathrm{C}(5)), 48.93(\mathrm{t}, \mathrm{C}(3)), 40.80(\mathrm{t}, \mathrm{C}(2)), 28.77(\mathrm{t}, \mathrm{C}(6))$. FAB-MS $m / z: 217\left[(\mathrm{M}+1)^{+}\right]$.

5-Hydroxy-5-hydroxymethyl-2,3,5,6-tetrahydro-7H-thiazolo[3,2-a]pyrimidin-7-one (16c)-A solution of 3c $(300 \mathrm{mg}, 1.63 \mathrm{mmol})$ in $5 \% \mathrm{HCl}(8 \mathrm{ml})$ was heated at $95-100{ }^{\circ} \mathrm{C}$ for $6 \mathrm{~h}$. The reaction mixture was worked up as described above, giving $190 \mathrm{mg}\left(57.7 \%, \mathrm{H}_{2} \mathrm{O}\right)$ of colorless prisms, mp $210-212{ }^{\circ} \mathrm{C}$. Anal. Calcd for $\mathrm{C}_{7} \mathrm{H}_{10} \mathrm{~N}_{2} \mathrm{O}_{3} \mathrm{~S}: \mathrm{C}$, 41.58; H, 4.98; N, 13.85. Found: C, 41.50; H, 4.85; N, 13.71. IR (KBr): 3395, $3190(\mathrm{OH}), 1710(\mathrm{sh}), 1695$ $(\mathrm{C}=\mathrm{O}) \mathrm{cm}^{-1}$. UV $\lambda_{\max }^{\text {ethal }} \mathrm{nm}(\log \varepsilon): 262(\mathrm{sh}, 3.45) .{ }^{1} \mathrm{H}-\mathrm{NMR}\left(270 \mathrm{MHz}: \mathrm{CDCl}_{3}\right.$ and 3 drops of $\mathrm{CF}_{3} \mathrm{COOH} \delta: 9.32$ $(1 \mathrm{H}, \mathrm{s}, \mathrm{OH}), 4.44(1 \mathrm{H}, \mathrm{ddd}, J=5.0,7.0,12.1 \mathrm{~Hz}, \mathrm{~N}-\mathrm{CH}), 3.96(1 \mathrm{H}, \mathrm{d}, J=12.6 \mathrm{~Hz}, \mathrm{C}(6)-\mathrm{H}), 3.80(1 \mathrm{H}, \mathrm{dt}, J=6.6,7.0$, $12.1 \mathrm{~Hz}, \mathrm{~N}-\mathrm{CH}), 3.78(1 \mathrm{H}, \mathrm{d}, J=12.6 \mathrm{~Hz}, \mathrm{C}(6)-\mathrm{H}), 3.3-3.15\left(2 \mathrm{H}, \mathrm{m}, \mathrm{S}-\mathrm{CH}_{2}\right), 3.23\left(2 \mathrm{H}, \mathrm{s}, \mathrm{O}-\mathrm{CH}_{2}\right) .{ }^{13} \mathrm{C}-\mathrm{NMR}$ (pyridine- $d_{5}$ ) $\delta: 169.4(\mathrm{~s}, \mathrm{C}(7)), 151.5$ (s, C(la)), $71.5(\mathrm{~s}, \mathrm{C}(5)), 69.0\left(\mathrm{t}, \mathrm{CH}_{2} \mathrm{OH}\right), 48.4(\mathrm{t}, \mathrm{C}(3)), 41.8(\mathrm{t}, \mathrm{C}(2))$ ) FAB-MS $m / z: 203\left[(\mathrm{M}+1)^{+}\right]$.

1-(2-Mercaptoethyl)-6-phenyl-2,4(1 H,3H)-pyrimidinedione (18)-A solution of $17(0.5 \mathrm{~g}, 2.17 \mathrm{mmol})$ in $5 \%$ $\mathrm{HCl}(10 \mathrm{ml})$ was refluxed for $4 \mathrm{~h}$. The reaction mixture was worked up as described above, giving $0.30 \mathrm{~g}(55.8 \%$, AcOEt) of colorless needles, mp $164-165^{\circ} \mathrm{C}$. Anal. Calcd for $\mathrm{C}_{12} \mathrm{H}_{12} \mathrm{~N}_{2} \mathrm{O}_{2} \mathrm{~S}: \mathrm{C}, 58.05 ; \mathrm{H}, 4.87 ; \mathrm{N}, 11.28$. Found: C, 58.35; H, 4.97; N, 11.06. IR (KBr): $2530(\mathrm{SH}), 1702(\mathrm{C}=\mathrm{O}) \mathrm{cm}^{-1}$. UV $i_{\max }^{\text {ethanol }} \mathrm{nm}(\log \varepsilon): 275(4.05) .{ }^{1} \mathrm{H}-\mathrm{NMR}$ $\left(\mathrm{CDCl}_{3}\right) \delta: 9.4\left(1 \mathrm{H}\right.$, br s, NH), 7.6-7.2 $\left(5 \mathrm{H}, \mathrm{m}, \mathrm{C}_{6} \mathrm{H}_{5}\right), 5.63(1 \mathrm{H}, \mathrm{s}, \mathrm{ring}), 3.83\left(2 \mathrm{H}, \mathrm{dd}, J=7.25,5.27 \mathrm{~Hz}, \mathrm{~N}-\mathrm{CH}_{2}\right)$, $2.63\left(2 \mathrm{H}, \mathrm{m}, \mathrm{S}-\mathrm{CH}_{2}\right), 1.19(1 \mathrm{H}, \mathrm{t}, J=8.8 \mathrm{~Hz}, \mathrm{SH})$.

3-(2-Mercaptoethyl)-6-methyl-2,4(1 $\mathbf{H , 3 H})$-pyrimidinedione (19)-A solution of $11(1.0 \mathrm{~g}, 5.95 \mathrm{mmol})$ in $5 \%$ $\mathrm{HCl}(20 \mathrm{ml})$ was refluxed for $2 \mathrm{~h}$. The reaction mixture was worked up as described above, giving $0.65 \mathrm{~g}\left(58.1 \%, \mathrm{H}_{2} \mathrm{O}\right)$ of colorless prisms, $\mathrm{mp} 203^{\circ} \mathrm{C}$. Anal. Calcd for $\mathrm{C}_{7} \mathrm{H}_{10} \mathrm{~N}_{2} \mathrm{O}_{2} \mathrm{~S} \cdot 1 / 10 \mathrm{H}_{2} \mathrm{O}: \mathrm{C}, 44.71 ; \mathrm{H}, 5.47 ; \mathrm{N}, 14.90$. Found: C, 44.76; $\mathrm{H}, 5.30 ; \mathrm{N}, 14.67$. IR (KBr): $2555(\mathrm{SH}), 1725,1705,1642(\mathrm{C}=\mathrm{O}) \mathrm{cm}^{-1}$. UV $\lambda_{\max }^{\text {ethal }} \mathrm{nm}(\log \varepsilon): 264(4.08)$. FAB-MS $m / z: 187\left[(\mathrm{M}+1)^{+}\right] .{ }^{1} \mathrm{H}-\mathrm{NMR}\left(\mathrm{CDCl}_{3}\right.$ and 5 drops of $\left.\mathrm{CF}_{3} \mathrm{COOH}\right) \delta: 10.37(1 \mathrm{H}, \mathrm{brs}, \mathrm{NH}), 5.91(1 \mathrm{H}, \mathrm{q}, J=0.88 \mathrm{~Hz}$, ring), $4.14\left(2 \mathrm{H}, \mathrm{dd}, J=7.03,5.71 \mathrm{~Hz}, \mathrm{~N}-\mathrm{CH}_{2}\right), 2.80\left(2 \mathrm{H}, \mathrm{m}, \mathrm{S}-\mathrm{CH}_{2}\right), 2.26\left(3 \mathrm{H}, \mathrm{d}, J=0.88 \mathrm{~Hz}, \mathrm{CCH}_{3}\right), 1.41(1 \mathrm{H}, \mathrm{t}, J=$ $\left.8.7 \mathrm{~Hz}, \mathrm{CH}_{2} \mathrm{~S} \underline{\mathrm{H}}\right) .{ }^{13} \mathrm{C}-\mathrm{NMR}$ (DMSO- $\left.d_{6}\right) \delta: 162.43(\mathrm{C}(4)), 151.27(\mathrm{C}(2$ or 6$)), 151.16(\mathrm{C}(6$ or 2$)), 98.18(\mathrm{C}(5)), 41.77$ $\left(\mathrm{CH}_{2} \mathrm{~N}\right), 21.02\left(\mathrm{CH}_{2} \mathrm{SH}\right), 17.93\left(\mathrm{CH}_{3}\right)$.

3,3'-Bis[6-methyl-1,2,3,4-tetrahydro-2,4-dioxopyrimidinyl]diethyldisulfide (20)-A solution of 11 (336 mg, $2.00 \mathrm{mmol})$ in $5 \% \mathrm{HCl}(30 \mathrm{ml})$ was heated at $95-100 \mathrm{C}$ for $7 \mathrm{~h}$. The reaction mixture was concentrated to dryness in vacuo. Water was added to the residue, and the mixture was evaporated to dryness in vacuo. The residue was treated with $5 \% \mathrm{NaHCO}_{3}$, and the insoluble crystalline mass was collected and recrystallized from $\mathrm{MeOH}$, giving $100 \mathrm{mg}$ $\left(26.6 \%\right.$ ) of colorless powder, mp $283-285^{\circ} \mathrm{C}$. Anal. Calcd for $\mathrm{C}_{14} \mathrm{H}_{18} \mathrm{~N}_{4} \mathrm{O}_{4} \mathrm{~S}_{2} \cdot 0.3 \mathrm{H}_{2} \mathrm{O}: \mathrm{C}, 44.74 ; \mathrm{H}, 4.99 ; \mathrm{N}, 14.91$. Found: C, 44.69; H, 4.95; N, 14.50. IR (KBr): $3550,3430(\mathrm{NH}), 1730,1707(\mathrm{sh}), 1645(\mathrm{C}=\mathrm{O}) \mathrm{cm}^{-1}$. UV $\lambda_{\max }^{\text {ethanol }} \mathrm{nm}$ $(\log \varepsilon): 263$ (4.36). FAB-MS $m / z: 371\left[(\mathrm{M}+1)^{+}\right] .{ }^{1} \mathrm{H}-\mathrm{NMR}\left(\mathrm{CDCl}_{3}\right.$ and 3 drops of $\left.\mathrm{CF}_{3} \mathrm{COOH}\right) \delta: 5.92(2 \mathrm{H}, \mathrm{s}, 2 \times \mathrm{ring})$, $4.33\left(4 \mathrm{H}, \mathrm{dd}, J=7.91,6.59 \mathrm{~Hz}, 2 \times \mathrm{N}-\mathrm{CH}_{2}\right), 2.97\left(4 \mathrm{H}, \mathrm{dd}, J=7.91,6.59 \mathrm{~Hz}, 2 \times \mathrm{S}-\mathrm{CH}_{2}\right), 2.28\left(6 \mathrm{H}, \mathrm{s}, 2 \times \mathrm{CH}_{3}\right)$.

Acknowledgement The authors are grateful to Mr. Y. Kinoshita, Kissei Pharmaceutical Industry, for measuring the ${ }^{1} \mathrm{H}-\mathrm{NMR}(270 \mathrm{MHz})$ spectra. Thanks are also due to Mrs. H. Mazume, Mr. M. Ohwatari, Mr. K. Inada, and Mr. N. Yamaguchi for elemental analysis, IR, UV, NMR, and mass spectra.

\section{References}

1) a) N. G. Pashkurov and V. S. Reznik, Khim. Geterotsikl. Soedin, 1968, 918 [Chem. Abstr., 71, 13088 (1969)]; b) 
G. R. Brown and W. R. Dyson, J. Chem. Soc. C, 1971, 1527.

2) a) E. Campaigne, J. C. Huffman, and T. P. Selby, J. Heterocycl. Chem., 16, 725 (1979); b) E. Campaigne, K. Folting, J. C. Huffman, and T. P. Selby, ibid., 18, 575 (1981).

3) M. Szajda and E. Wyrzykiewicz, Pol. J. Chem., 57, 1027 (1983).

4) G. Shaw and R. N. Warrener, J. Chem. Soc., 1959, 50.

5) a) S. Giambrone, Bull. Sci. Fac. Chim. Ind. Bologna, 11, 86 (1953) [Chem. Abstr., 49, 4662 (1955)]; b) V. Škarić, D. Škarić, and A. Čižmek, J. Chem. Soc., Perkin Trans. 1, 1984, 2221; c) M. Mizutani, Y. Sanemitsu, Y. Tamura, and Z. Yoshida, J. Org. Chem., 50, 764 (1985).

6) T. Kato, N. Katagiri, U. Izumi, Y. Miura, T. Yamazaki, and Y. Hirai, Heterocycles, 15, 399 (1981).

7) a) M. Seperic, Ger Offen. 2140601 [Chem. Abstr., 77, 5516 (1972)]; b) J. Baetz, Brit. Patent 1334628 [Chem. Abstr., 80, 48028 (1974)]; c) SEPERIC, Fr. Demande, 2173668 [Chem. Abstr., 80, 96015 (1974)]; d) SEPERIC, Neth. Appl. 7600885 [Chem. Abstr., 87, 85046 (1977)]; e) SEPERIC, Bel. 778911 [Chem. Abstr., 80, 48028 (1974)].

8) K. D. Kampe, Angew. Chem., 94, 543 (1982).

9) H. N. Al-Jallo and M. A. Muniem, J. Heterocycl. Chem., 15, 849 (1978).

10) R. A. Glennon, R. G. Bass, and E. Schubert, J. Heterocycl. Chem., 16, 903 (1979).

11) a) N. Okamura, T. Toru, T. Tanaka, K. Watanabe, K. Bannai, S. Kurozumi, T. Naruchi, and K. Kamoriya, Eur. Pat. Appl., EP 49902 [Chem. Abstr., 97, 92311 (1982)]; b) Teijin Ltd., Jpn. Kokai Tokkyo Koho JP 83 24590 [Chem. Abstr., 98, 215611 (1983)]; c) Teijin Ltd., Jpn. Kokai Tokkyo Koho JP 8488491 [Chem. Abstr., 101, 230562 (1984)].

12) R. N. Warrener and E. N. Cain, Aust. J. Chem., 24, 785 (1971).

13) D. W. Dunwell and D. Evans, J. Chem. Soc. C, 1971, 2094.

14) K. Tabei, E. Kawashima, T. Takeda, and T. Kato, Chem. Pharm. Bull., 30, 3987 (1982).

15) K. Hirota, Y. Yamada, Y. Kitade, and S. Senda, J. Chem. Soc., Perkin Trans. I, 1981, 2943.

16) E. Falch and T. Natvig, Acta Chem. Scand., 24, 1423 (1970).

17) T. Kinoshita, H. Tanaka, and S. Furukawa, Chem. Pharm. Bull., 34, 1809 (1986). 